\section{Military Technical College Kobry El-Kobbah, Cairo, Egypt}

\author{
$6^{\text {th }}$ International Conference \\ on Electrical Engineering \\ ICEENG 2008
}

\title{
Performance enhancement of Yagi-Uda antenna using bulk micromachining technique
}

By

Walaa wahba ibrahim*

A.Abd-El-Nazir.Ahmed*
A. Hafez. Zaki*

M.Abd-El-Azez.Mitkes*
Abd-El-Maged Allam*

\section{Abstract:}

In this paper, silicon micromachining has been applied to enhance the antenna performance. The work is devoted to diminish the effect of propagation of higher order modes in substrates with high permittivity in printed antennas; hence one can increase the antenna bandwidth. The process of micromachining can remove the substrate of the silicon material of a substrate under some selected elements to enhance the antenna performance. after etching, the new given structure is effectively enhance the performance for Yagi Uda printed array operating at $\mathrm{f}=10 \mathrm{GHz}$.

\section{Keywords:}

Silicon micromachining, Micromachined antennas. 


\section{Introduction:}

In keeping with the trend towards using higher frequencies in communications, (e.g., space applications at $30 \mathrm{GHz}$, local multi point distribution systems (LMDS) at $28 \mathrm{GHz}$ and wireless local area networks (WLANs) at around $60 \mathrm{GHz}$ ) the antenna technology needs to meet new requirements [1]. On the other hand; as more components are integrated into circuits at the chip level, semiconductor substrates must be used for antennas. It is difficult to achieve this issue by the traditional techniques of fabrication; this is because of the diametrically opposite physical processes needed for the circuits and antennas [2]. Circuits require closely bound fields and hence thin substrates of high permittivity are preferred. Microstrip patch antennas need to radiate and hence the requirement is for loosely bound fields with lot of fringing and it requires thick substrates of low permittivity. The big challenge is to satisfy both the requirements on the same wafer.

Micromachining the semiconductor substrate to produce an air cavity under the patch has been proposed as one of the solutions to overcome the problem of integrating both antenna and electronic components on the same substrate [3,4,5]. The advantages of micromachining in microstrip patch antennas are multifaceted. This approach can effectively reduce the effective dielectric constant of the substrate by selectively removing material (e.g., underneath the patch) and thus improve its bandwidth and radiation efficiency. The undesirable effects of surface waves, which are prominent in high $\varepsilon_{\mathrm{r}}$ substrates at millimeter wave frequencies can be mitigated by Micromachining process.

Emerging wireless communication and sensor applications in the RF, Microwave, and millimeter wave regimes require low loss and high performance antennas. A good antenna design for these new circumstances is not just simply scaling classical antenna to different frequencies, but also to meet different operating requirements. In many applications, an end-fire radiation pattern is needed to have the electromagnetic wave propagate along the supporting substrate. To generate this pattern, Yagi-Uda antenna can be used [6,7]. It has been used for almost eight decades since Uda and Yagi first investigated it in 1920's. It consists of a driven element, a reflector and several directors. Only the driven element is directly fed by source while the others are parasitically coupled. Since only a few elements for a Yagi-Uda array needs to be fed directly, the introduced simplicity has made it find many applications including outdoor TV signal receiving. 


\section{A Vertical W-band Surface-Micromachined Yagi-Uda Antenna}

The traditional Yagi-Uda antennas used for HF and UHF application are usually built by several metal rods and not well suited for integration. A printed vertical version was first proposed by Y. Yoon [8] as shown in Fig 1. Unlike the classical wire Yagi-Uda used in UHF applications, Monopoles instead of dipoles are used due to the existence of ground plane on top of wafer. According to the mirror theory in the upper half space; it is equivalent to a dipole Yagi-Uda array. It consists of one driving monopole, one reflector and several directors. The coplanar waveguide (CPW) feeding is connected with driving monopole. The antenna is designed at $100 \mathrm{GHz}$, with spacing between adjacent elements is set to $480 \mu \mathrm{m}$. The heights for reflector, driving monopole and directors are $800 \mu \mathrm{m}, 715 \mu \mathrm{m}, 560 \mu \mathrm{m}$, respectively. The predicted return loss for a 5element Yagi-Uda antenna on glass substrate is shown in Fig.2.

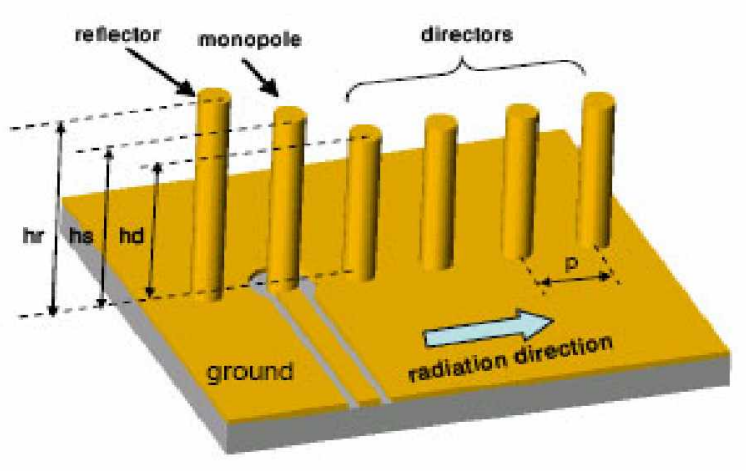

Figure (1): 5-elelments W-band yagi-uda antenna on glass substrate

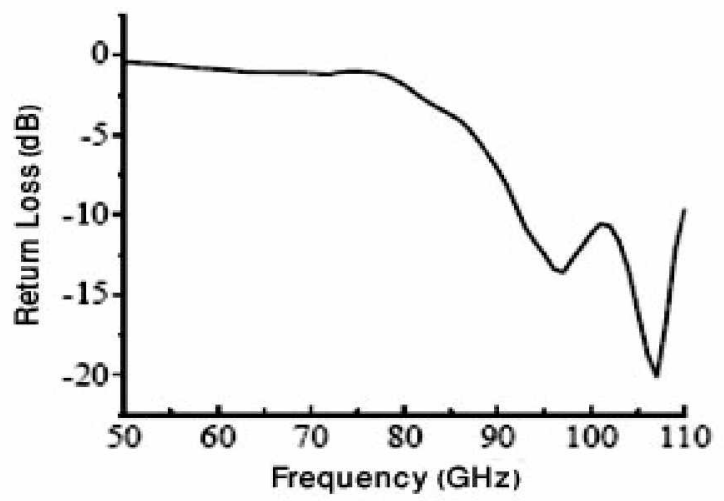

Figure (2): Return loss of Vertical W-band Yagi-Uda Antenna on glass substrate

From Fig.2, it is clear that, although the antenna is designed at $100 \mathrm{GHz}$, the resonance frequency at the simulated result is shifted; this is due to the placing of the array over a semiconductor substrate not on free space, also the feed position due to the using of CPW led to this shift on the resonance frequency.

\section{Scaling the design of the vertical Yagi-Uda antenna:}

The design is scaled to $10 \mathrm{GHz}$ (as shown in Fig.3) to match the fabrication and measuring capabilities, the dimensions after scaling are: spacing between adjacent elements is set to $3.8 \mathrm{~mm}$, and the height for reflector, driving monopole and directors are $8 \mathrm{~mm}, 7.15 \mathrm{~mm}, 5.6 \mathrm{~mm}$ respectively. Electromagnetic simulation have been 
performed using Ansoft high frequency structure simulator (HFSS) 10.1, The predicted return loss for a 5-element Yagi-Uda antenna (after scaling) is plotted in Fig.4, also radiation pattern is shown in Fig.5.

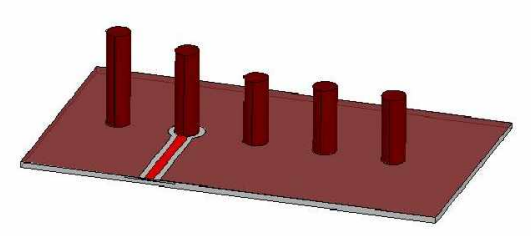

Figure (3): 5-elelments Yagi-Uda antenna on silicon substrate

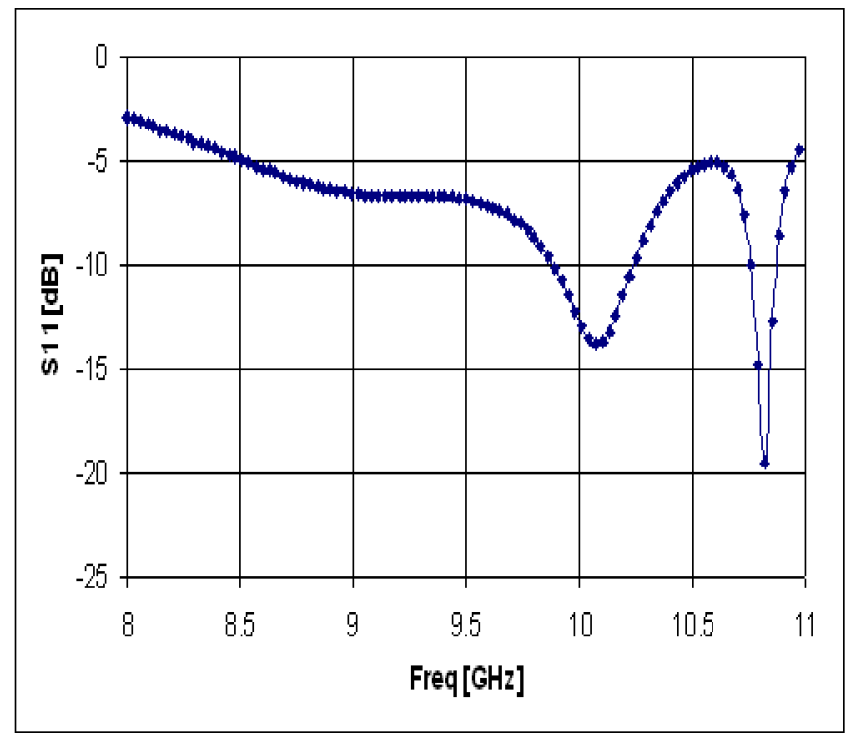

Figure (4): Simulated performance of 5element array :Return loss

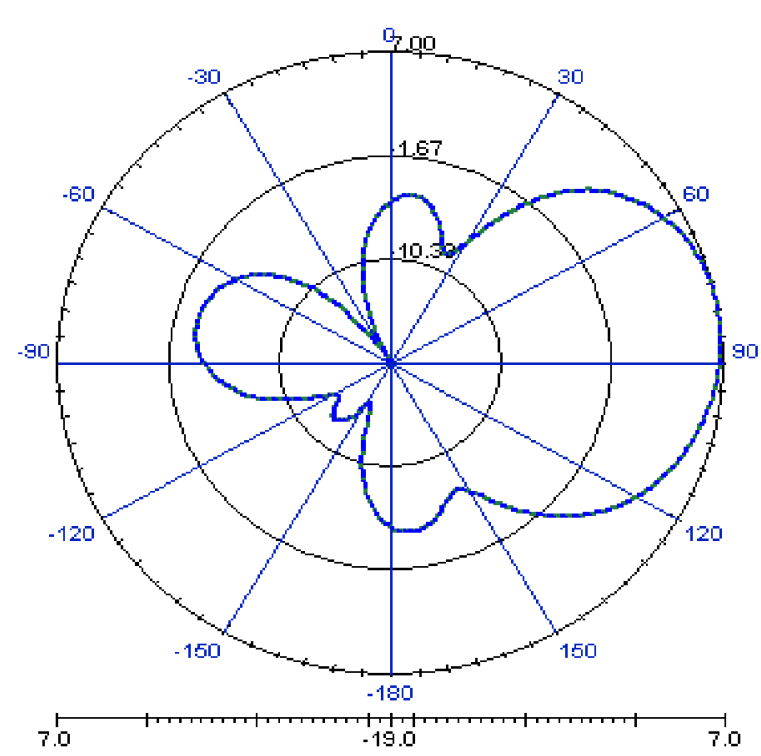

Figure (5): Simulated performance of 5element array $:$ Radiation pattern

\section{Enhancement using micromachining:}

This work utilizes the concept of bulk micromachining techniques; the material used in our designs was a silicon substrate with relative permittivity $\left(\varepsilon_{\mathrm{r}}\right)$ of 11.9 , conductivity $(\sigma)$ of $100 \mathrm{~S} / \mathrm{m}$ and thickness of $500 \mu \mathrm{m}$.

In order to achieve a cavity in the silicon substrate, an appropriate silicon etchant is used. Potassium hydroxide $(\mathrm{KOH})$ is a wet silicon etchant which attacks silicon preferentially in the $\langle 100\rangle$ plane, producing a characteristic anisotropic V-etch, with 
sidewalls that form a $54.7^{\circ}$ angle with the surface ( $35.3^{\circ}$ from the normal). The $\mathrm{KOH}$ process takes typically 1 hour for $40 \mu \mathrm{m}$ etch.

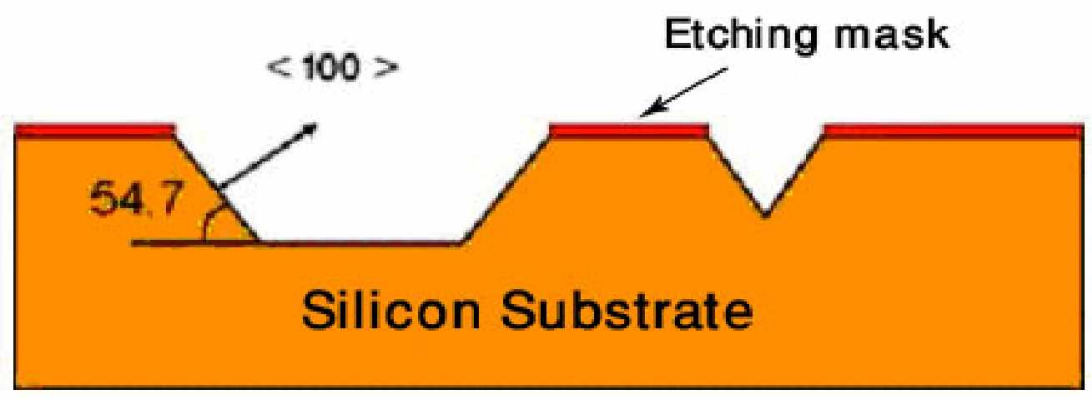

Figure (6): Anisotropic etching of silicon substrate

\section{The design optimization}

One of the most important steps in the design of the printed antenna on a micromachined substrate is to determine the best diameter and the height of the hole under the antenna element that gives the best performance. This optimization can be done by using the parametric analysis which allows making a sweep for $S_{11}$ parameters over different diameter and thickness of the hole. the simulation indicate that the best value for diameter and thickness are $1.8 \mathrm{~mm}, 250 \mu \mathrm{m}$ respectively as shown in Fig.8.The antenna is resimulated again with holes in the silicon substrate underneath each element with the optimized diameter and thickness to examine the effect of silicon etching. The predicted return loss for a 5-element Yagi-Uda antenna (after etching) is plotted in Fig.9; with its radiation pattern shown in Fig.10.

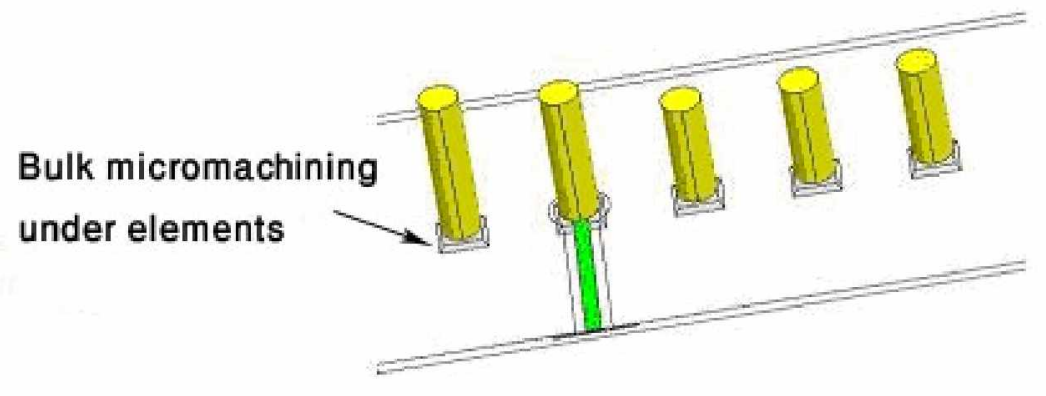

Figure (7): 5-elements antenna over etched substrate 


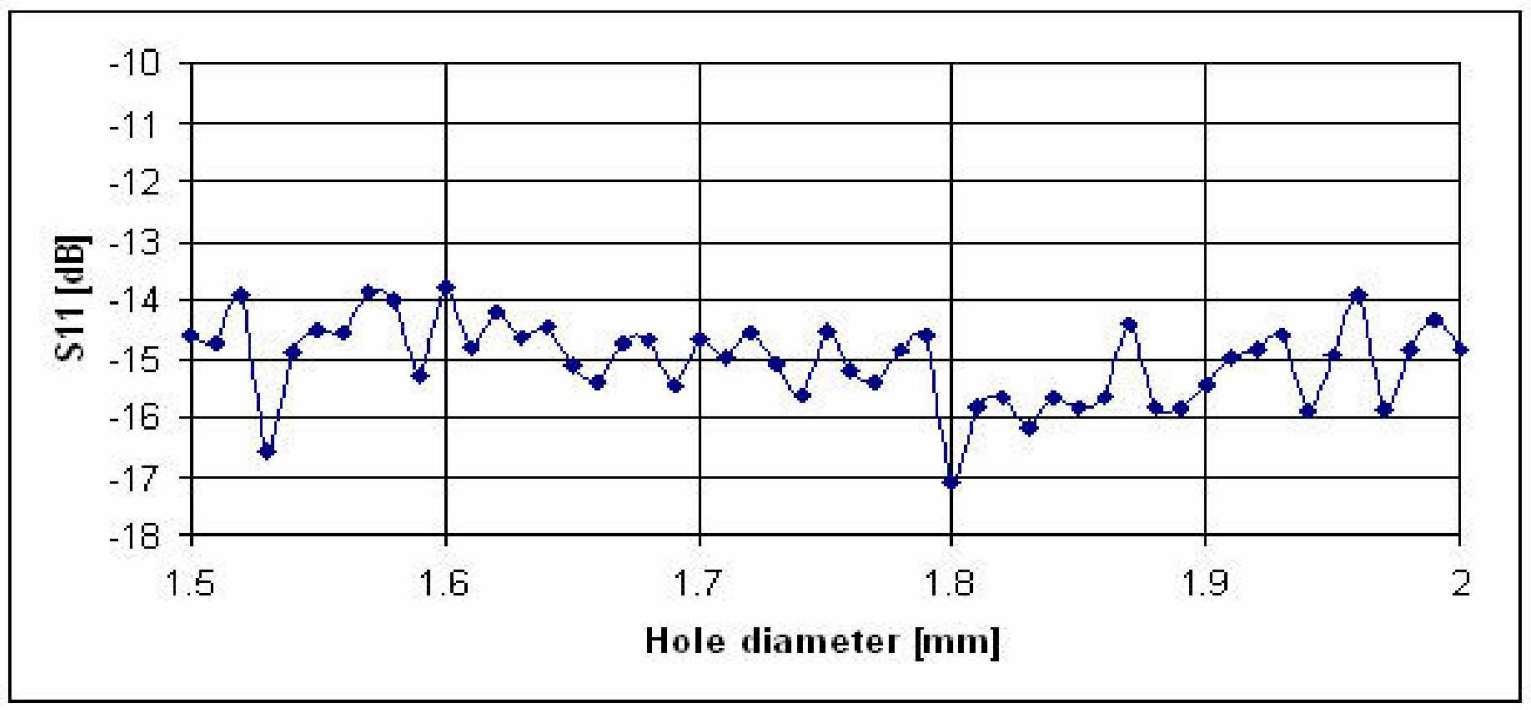

Figure (8): Variation of $\left(S_{11}\right)$ over hole diameter

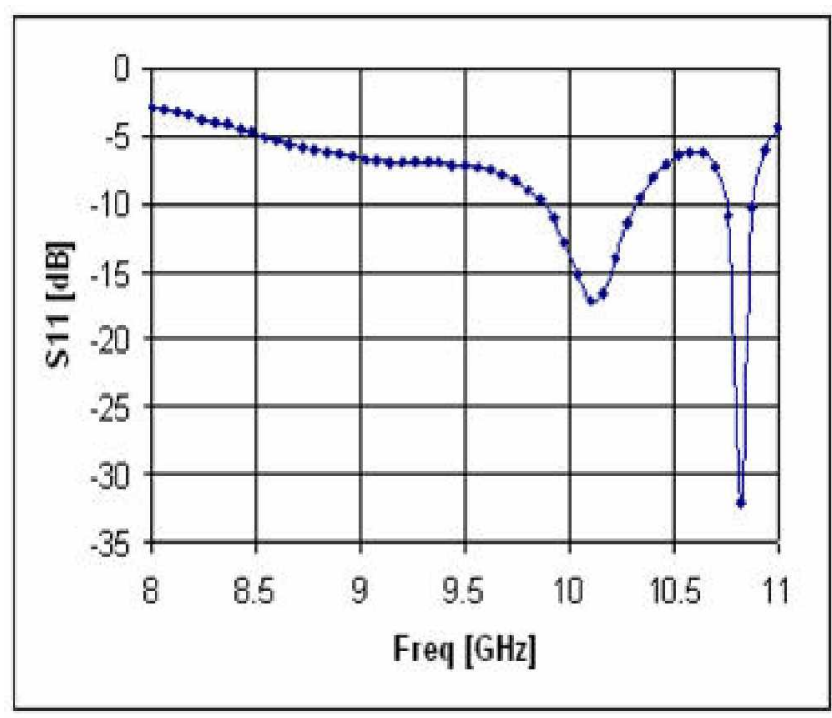

Figure (9): Simulated performance of 5 -element array:Return loss

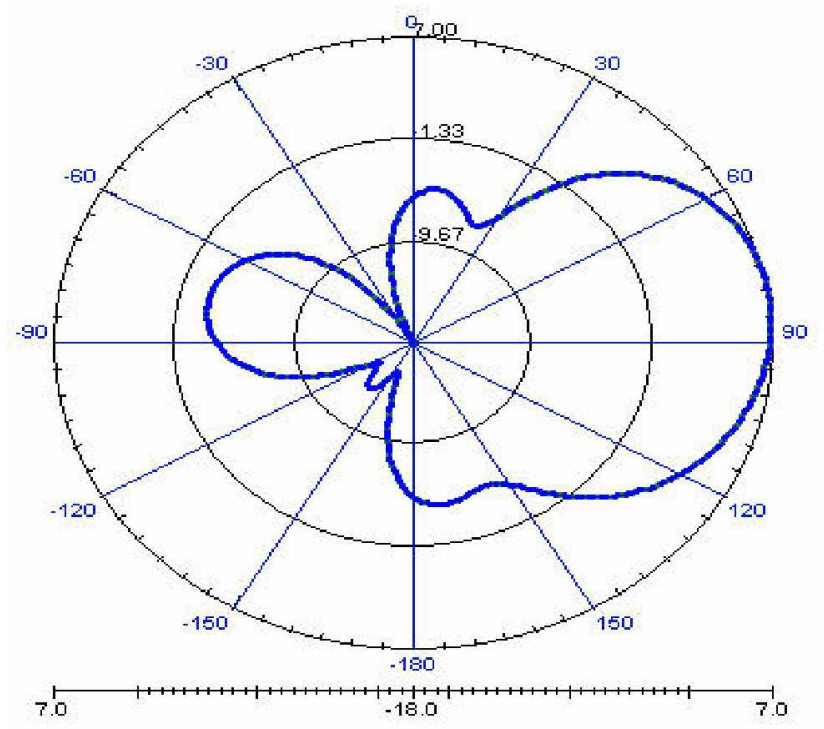

Figure (10): Simulated performance of 5-element array :Radiation pattern

Fig.9 shows that the simulated return loss at the case of using bulk micromachining technique is $-17.1 \mathrm{~dB}$ which is better than the ordinary design $(-13 \mathrm{~dB})$, this is due to the fact that the relative permittivity $\left(\varepsilon_{\mathrm{r}}\right)$ of the silicon substrate is decreased after etching process. to determine the value of effective permittivity ( $\left.\varepsilon_{\text {reff }}\right)$ that led to this enhancement in the return loss, the parametric analysis is used again and give the value of $\left(\varepsilon_{\text {reff }}\right)=11.23$. 
Conclusion : This proposed design has low surface-wave effects due to its air extruded geometry when compared with conventional planar devices. The driving monopole is fed by a co-fabricated CPW line facilitating easy connectivity of the antenna to other components as well as easy fabrication. It's clear that the bulk micromachining technique has led to enhance both return loss $(-4.1 \mathrm{~dB})$ and band width $(1.6 \%)$.

\section{References:}

[1] Holger H. Meinel, "Millimeter-Wave Technology Advances Since 1985 and Future Trends", IEEE Trans. On microwave theory and techniques. Vol 39, NO 5. May 1991.

[2] Pozar D.M., "Considerations for millimeter wave printed antennas", IEEE Trans. on Antennas and Propagation, Vol 31, pp. 740-747, 1993.

[3] Yook J.G. and Katehi L.P.B., "Micromachined Microstrip Patch Antenna With Controlled Mutual Coupling and Surface Waves", IEEE Trans. on Antennas and Propagation, Vol 49, pp. 1282-1289, 2001.

[4] Alan Courtay, Gildas P. Gauthier and Gabriel M. Rebeiz, "Microstrip Antennas on Localized Micromachined Dielectric Substrates ", IEEE Trans. on Antennas and Propagation, [1996].

[5] P. M. Mendes, H. Correia, "Efficiency Improvement of a Chip-Size Antenna for Wireless Microsystems Using Bulk-Micromachining Technology ".

[6] C. Balanis, Antenna Theory: Analysis and Design, 2nd ed. New York: Wiley, 1997.

[7] GARY A. THIELE, "Analysis of Yagi-Uda Type Antennas", IEEE Trans. on Antennas and Propagation, VOL. AP-17, NO. 1, January 1969.

[8] Y. Yoon, Bo Pan, J. Papapolymerou, M. M. Tentzeris and M. G. Allen "A Vertical W-band Surface-Micromachined Yagi-Uda Antenna". 